\title{
SPECTRAL-SPATIAL CLASSIFICATION OF HYPERSPECTRAL IMAGERY USING A HYBRID FRAMEWORK
}

\author{
D. Akbari ${ }^{1}$, M. Moradizadeh ${ }^{2}$ \\ ${ }^{1}$ Dept. of Surveying and Geomatics Engineering, College of Engineering, University of Zabol, Zabol, Iran- davoodakbari@ut.ac.ir \\ ${ }^{2}$ Dept. of Geomatics, Faculty of Civil and Transportation Engineering, University of Isfahan, Isfahan, Iran- \\ m.moradizadeh@eng.ui.ac.ir
}

Commission VI, WG VI/4

KEY WORDS: Remote sensing, Hyperspectral image, neural network, Marker selection

\begin{abstract}
:
Hyperspectral Images are worthwhile data for many processing algorithms (e.g. Dimensionality Reduction, Target Detection, Change Detection, Classification and Unmixing). Classification is a key issue in processing hyperspectral images. Spectral-identificationbased algorithms are sensitive to spectral variability and noise in acquisition. There are many algorithms for classification. This paper describes a new framework for classification of hyperspectral images, based on both spectral and spatial information. The spatial information is obtained by an enhanced Marker-based Hierarchical Segmentation (MHS) algorithm. The hyperspectral data is first fed into the Multi-Layer Perceptron (MLP) neural network classification algorithm. Then, the MHS algorithm is applied in order to increase the accuracy of less-accurately classified land-cover types. In the proposed approach, the markers are extracted from the classification maps obtained by MLP and Support Vector Machines (SVM) classifiers. Experimental results on Quebec City hyperspectral dataset, demonstrate that the proposed approach achieves approximately $9 \%$ and $5 \%$ better overall accuracy than the MLP and the original MHS algorithms respectively.
\end{abstract}

\section{INTRODUCTION}

Hyperspectral imagery has been widely investigated for landcover classification due to its broad coverage of wavelength and high spectral sampling rate. Among the many studies that have been published on this topic, two main categories of techniques have been established: the spectral (i.e., pixel-based) techniques and the spectral-spatial (i.e., object-based) techniques. The pixel-based classification methods are often unable to accurately differentiate between some classes with high spectral similarity. This is mainly because they employ only the spectral information in order to identify different land-cover types. Consequently, methods that can exploit the spatial information are essential for more accurate classification results (Carleer and Wolff, 2006; Shackelford and Davis, 2003).Many researchers have demonstrated that the use of spectral-spatial information improves the classification results, compared to the use of spectral data alone, in hyperspectral imagery (Argüello and Heras, 2015; Blaschke et al., 2014; Fauvel et al., 2012; Huang and Zhang, 2011; Negri et al., 2014; Paneque-Gálvez et al., 2013; Tarabalka et al., 2010).In the early studies on these methods, the spectral information from the neighborhoods is extracted by either a fixed size window (Camps-Valls et al., 2006) or morphological profiles (Fauvel et al., 2008), and used for classifying and labeling of image pixels.

Segmentation techniques are powerful means for defining the spatial dependencies among the pixels and for finding the homogeneous regions in an image (Gonzalez and Woods, 2002). Among the various methods of segmentation, the hierarchical segmentation method is state-of-the-art for hyperspectral image analysis (Tilton,2003). An alternative way in order to improve the accuracy of segmentation is performing a marker-based technique (Gonzalez and Woods, 2002; Soille, 2003). In this approach for each spatial object of the image, one or several pixels are selected as seed or marker. The selected markers then grow and create a unique region in the segmentation map. Marker-based segmentation considerably decreases the over-segmentation, and as a result, leads to more reliable accuracies(Soille, 2003).

In (Tarabalka et al., 2011) an efficient approach was proposed for spectral-spatial classification using the Marker-based Hierarchical Segmentation (MHS) grown from automatically selected markers. It uses a pixel-wise SVM classification, in order to select pixels with the highest probability estimate to each class, as markers. In this framework, a connected components labeling is applied on the classification map. Then, if a region is large enough, its marker is determined as the $\mathrm{P} \%$ of pixels within this region with the highest probability estimates. Otherwise, it should lead to a marker only if it is very reliable. A potential marker is formed by pixels with estimated probability higher than a defined threshold. The disadvantage of this approach is that the selection of the markers strongly depends on the performances of the selected pixel-wise classifier.

In this paper, a modified spectral-spatial classification approach is proposed for improving the spectral-spatial classification of hyperspectral images. In the proposed approach, the MLP neural network pixel-based algorithm is, first, used to classify the hyperspectral images. Afterwards, for classes with low accuracy, the enhanced MHS spectral-spatial algorithm is used to improve their accuracies. In the enhanced MHS algorithm, for selecting markers, the outputs of SVM and MLP classifiers are combined using the estimated class probability for each pixel. That is, each pixel is labeled to its corresponding class with maximum probability estimation of two classifiers. Then, the most reliable labeled pixels are selected as the markers. 


\section{THE PROPOSED ALGORITHM}

In the proposed framework, the hyperspectral image is first classified using MLP neural network algorithm. Then, the error rate for each class is computed as:

$$
E_{\mathrm{r}}=1-P_{\mathrm{A}}
$$

Where $P_{\mathrm{A}}$ is class-specific producer's accuracy. In the classification procedure, the high error rate of certain classes is not only an index of low accuracy between the set of classes, but also depends on the population of each class. Therefore, a classification measure, named $\delta$,can be defined for each class $i$ as follows:

$$
\delta_{i}=\frac{E_{i} \times P_{i}}{\max _{j=1 . . N}\left(E_{j} \times P_{j}\right)}
$$

Where $E_{i}$ and $P_{i}$ are, respectively, the error rate and the population size for class $i$, and $N$ is the number of classes. In this study, class $i$ has low accuracy if $\delta_{i} \geq 0.6$. The above value has been estimated by trial and error. The flowchart of the proposed framework is presented in Figure 1.

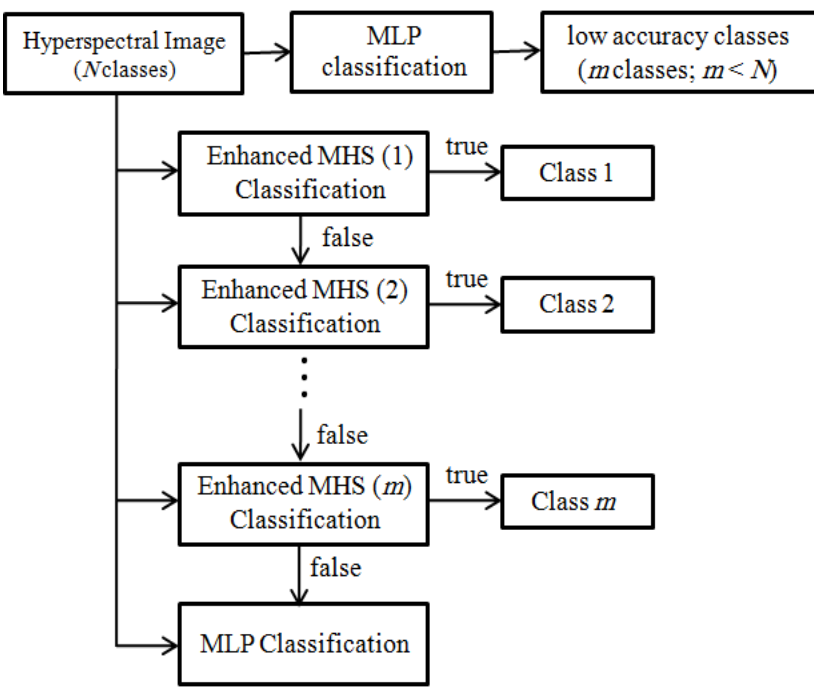

Figure 1. Schema of the proposed framework.

In proposed algorithm, the labeling of each pixel is first decided using the Enhanced $\mathrm{MHS}_{1}$ algorithm. Enhanced $\mathrm{MHS}_{1}$ is used in order to classify the image into two categories: a class with $\delta_{i}$ maximum value and the rest of classes. If the answer is negative, the pixel label can be found using the Enhanced $\mathrm{MHS}_{2}$ algorithm. Enhanced $\mathrm{MHS}_{2}$ is used to improve the class with $\delta_{i}$ value less than class of Enhanced $\mathrm{MHS}_{1}$ algorithm. This decision making process is continued using other Enhanced MHS algorithms until the answer is negative for the pixel label which is determined by MLP algorithm.

In Enhanced $\mathrm{MHS}_{\mathrm{i}}(\mathrm{i}=1, \ldots, \mathrm{m})$ classification used in this study, the outputs of SVM and MLP classifiers are combined using the estimated class probability for each pixel. The classification probabilities for each pixel $\mathrm{x}$ is given by

$$
P=\left\{p_{k}=p\left(L_{x}=k \mid x\right), k=1, \ldots, K\right\}
$$

Where, $\mathrm{L}_{\mathrm{x}}$ is the class label and $\mathrm{K}$ is the number of classes. For this purpose, pair-wise class probabilities $r_{i j} \approx p\left(L_{x}=i \mid L_{x}=\right.$ iorj, $\mathrm{x}$ ) are first estimated, and the probabilities in (3) are computed as described in (Wu et al., 2004). Then, a probability map is constructed by assigning the maximum probability estimation $\max \left(\mathrm{p}_{\mathrm{k}}\right), \mathrm{k}=1, \ldots, \mathrm{K}$, to each pixel. Finally, to combine the SVM and the MLP classification maps, after providing probability maps of both classifiers, each pixel is labeled to its corresponding class of classifiers which that classifier has maximum probability estimation. Lastly, the most reliable labeled pixels are selected as markers.

\section{EXPERIMENTAL RESULTS AND DISCUSSIONS}

\subsection{Hyperspectral Data}

To evaluate the proposed approach, the Quebec City hyperspectral dataset was selected. It was acquired by HyperCam LWIR over Quebec City. In the Quebec City dataset, the image pixel values represent the radiance values in the scene. Therefore, it requires atmospheric correction to perform the classification of images. ENVI's Thermal Atmospheric Correction algorithm was applied on this dataset. This dataset covers a spectral range of 7.8 to $12.5 \mu \mathrm{m}$. The scene has spatial dimensions of 564 columns and 795 rows, and a ground pixel size of $1 \mathrm{~m}$. The number of spectral bands is 84 . The reference data includes seven major classes: Road, Trees, Blue Roof, Gray Roof, Concrete Roof and Vegetation. For each of the classes, we randomly chose around $20 \%$ of the labeled samples for training and used the other $80 \%$ for testing.

\subsection{Experimental results}

In this study, a MLP classifier with three hidden layers including 4, 6, and 8 neurons is used. The evolution of the MLP performance is done using 600 iterations. In the MLP classification, as mentioned in section 2 , class $i$ has low accuracy if $\delta_{i} \geq 0.6$

In addition, the enhanced Marker-based Hierarchical Segmentation (MHS) method was applied in order to increase the accuracy of less-accurately classified land-cover types. For the SVM classifier, the Gaussian radial basis function (RBF), as kernel, is used (Camps-Valls and Bruzzone, 2005). The RBF kernel's parameters, i.e. $C$ and $\gamma$, are chosen by a five-fold cross validation. They are $C=86$ and $\gamma=0.3$. To create a map of markers, after combining the outputs of SVM and MLP classifiers using the estimated class probability for each pixel, labelling of connected components is performed using the eightneighbourhood connectivity. For each connected component, if it contains more than 40 pixels, $9 \%$ of its pixels with the highest estimated probability are selected as the marker for this component. Otherwise, the region marker is formed by the pixels with estimated probability higher than a threshold $\tau$. The threshold $\tau$ is equal to the lowest probability within the highest $6 \%$ of the probabilities for the whole image.

In order to compare the results of the proposed framework, we have implemented independently MLP, original-MHS and enhanced-MHS algorithms. The accuracies of the classification maps are generally assessed by computing the confusion matrix using the reference data.

Figure 2 shows the classification maps obtained by different methods and reference data. We can see from Figure 2 that by incorporating the spatial information, the proposed algorithm leads to much smoother classification maps when compared with the maps obtained by other methods (see Figure 2(d)).In addition, the enhanced-MHS is far less noisy in compared to the MLP and original-MHS. 


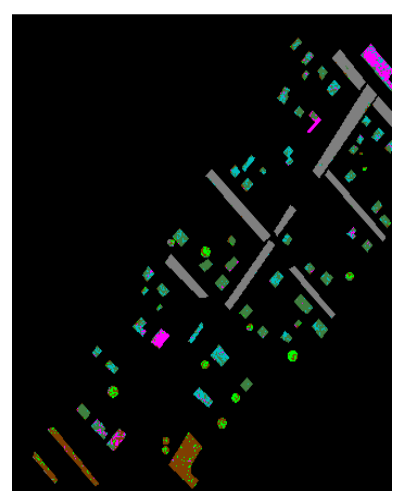

(a)

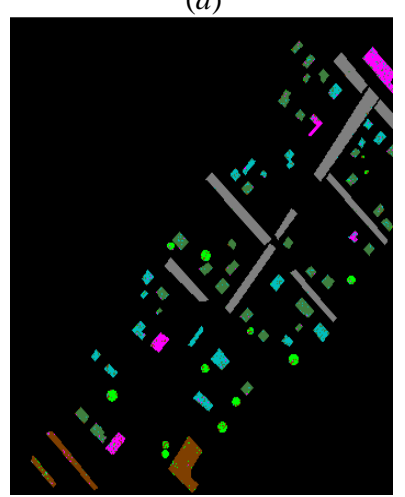

(c)

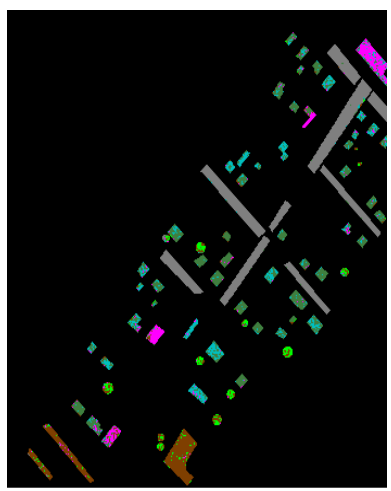

(b)

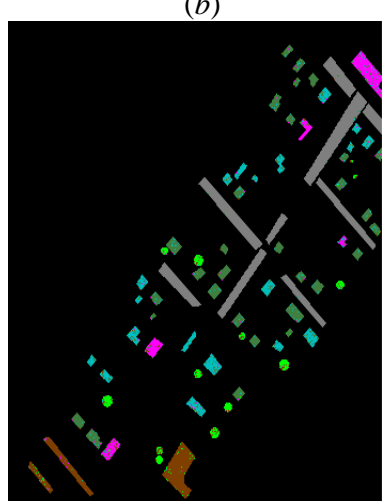

(d)

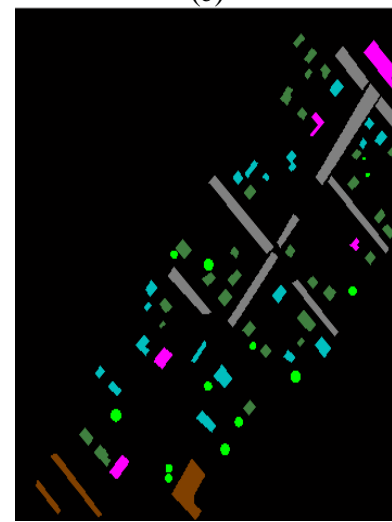

$(e)$

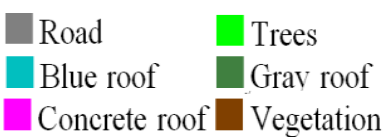

Figure 2. (a) MLP Classification map, (b) original-MHS classification map, $(c)$ enhanced-MHS Classification map, $(d)$ proposed Classification map, (e) Reference data.

Table 1 lists the classification accuracy rates obtained on the Quebec City dataset. We can see that Road, Trees, Gray Roof and Vegetation classes have a proposed parameter $\left(\delta_{i}\right)$ greater than 0.6, i.e. they have a low accuracy among the existing classes. For this dataset, the importance of including the spatial information is also evident, as it leads to an increase in accuracy. The OA obtained is higher, by about $9 \%, 5 \%$ and $2 \%$, respectively, than the accuracies of MLP, original-MHS and enhanced-MHS.

As Table 1 demonstrates, all of the class-specific producer's accuracies are considerably increased by the proposed approach when compared to MLP algorithm. Also, the enhanced-MHS algorithm in all classes except class Blue Roof is more accurate than the original-MHS algorithm. This decrease in accuracy can be due to the high dispersion and the low concentration of Blue Roof class in the image.

\begin{tabular}{cccccc}
\hline & MLP & $\delta_{i}$ & $\begin{array}{c}\text { original- } \\
\text { MHS }\end{array}$ & $\begin{array}{c}\text { enhanced- } \\
\text { MHS }\end{array}$ & $\begin{array}{c}\text { Proposed } \\
\text { algorithm }\end{array}$ \\
\hline OA $(\%)$ & 85.2 & - & 89.5 & 92.3 & $\mathbf{9 4 . 5}$ \\
$\kappa(\%)$ & 80.6 & - & 86.8 & 88.7 & $\mathbf{9 1 . 0}$ \\
\hline Road & 79.1 & $\mathbf{0 . 6 5}$ & 85.9 & 89.4 & $\mathbf{8 9 . 9}$ \\
Trees & 89.2 & $\mathbf{0 . 8 7}$ & 93.7 & 94.8 & $\mathbf{9 6 . 5}$ \\
Blue Roof & 89.6 & 0.25 & 89.7 & 89.6 & $\mathbf{9 1 . 3}$ \\
Gray Roof & 74.1 & $\mathbf{1}$ & 91.9 & 92.5 & $\mathbf{9 4 . 9}$ \\
Concrete & 87.5 & 0.39 & 89.5 & 92.3 & $\mathbf{9 5 . 1}$ \\
Roof & 90.2 & $\mathbf{0 . 7 5}$ & 90.2 & 91.5 & $\mathbf{9 3 . 3}$ \\
Vegetation &
\end{tabular}

Table 1.The classification accuracies obtained on the Quebec City dataset.

Table 2 shows the implementation time of the algorithms used. As it is seen, the implementation time of the proposed algorithm is close to that of enhanced-MHS algorithm. The reason is that the proposed approach is performed in parallel.

\begin{tabular}{ccccc}
\hline & MLP & $\begin{array}{c}\text { original- } \\
\text { MHS }\end{array}$ & $\begin{array}{c}\text { enhanced- } \\
\text { MHS }\end{array}$ & $\begin{array}{c}\text { Proposed } \\
\text { algorithm }\end{array}$ \\
\hline $\begin{array}{c}\text { Implementation } \\
\text { Time (Sec.) }\end{array}$ & 30 & 93 & 121 & 147 \\
\hline
\end{tabular}

Table 2.The implementation time of the algorithms used.

\section{CONCLUSION}

In this paper, a framework for the spectral-spatial classification of hyperspectral images has been proposed. In the proposed framework, the hyperspectral image is, first, classified using the MLP algorithm. Afterwards, the enhanced MHS spectral-spatial algorithm is used to improve the accuracy of low accuracy classes. In this algorithm, the markers are selected using the maps obtained from the combination of SVM and MLP classifications. The results demonstrate that the proposed algorithm generally a) improves the classification accuracy rates when compared to the classic MLP algorithm and the original MHS method, and b) provides classification maps with homogeneous regions. It is thus evident that spatial information for classification is very important.

The proposed approach has a drawback similar to almost all spectral-spatial techniques: it produces a smooth classification map in comparison to the pixel-wise classifications. Therefore, it risks impairing results near the borders between regions, where mixed pixels are often encountered. Spectral unmixing techniques can be used for accurate analysis of border regions.

\section{ACKNOWLEDGMENTS}

The authors would like to thank Telops Inc. (Québec, Canada) for providing the hyperspectral dataset used in this research.

\section{REFERENCES}

Argüello, F., and Heras, D.B., 2015. ELM-based spectralspatial classification of hyperspectral images using extended morphological profiles and composite feature mappings. International Journal of Remote Sensing, 36(2), 645-664.

Blaschke, T., Hay, G.J., Kelly, M., Lang, S., Hofmann, P., and Addink, E., 2014. Geographic Object-Based Image Analysis Towards a new paradigm. ISPRS Journal of Photogrammetry and Remote Sensing,87, 180-191.

Camps-Valls, G., and Bruzzone, L., 2005. Kernel-based methods for hyperspectral image classification. IEEE Translation Geoscience Remote Sensing, 43, 1351-1362. 
Camps-Valls, G., Gomez-Chova, L., Munoz-Mari, J., VilaFrances, J., and Calpe-Maravilla, J., 2006. Composite kernels for hyperspectral image classification. IEEE Geoscience and Remote Sensing Letters, 3, 93-97.

Carleer, A.P., and Wolff, E., 2006. Urban land cover multi-level region-based classification of VHR data by selecting relevant features. International Journal of Remote Sensing, 27(6), 10351051.

Fauvel, M., Chanussot, J., andBenediktsson, J.A., 2012. A spatial-spectralkernel-based approach for the classification of remote sensing images.Pattern Recognition,45, 381-392.

Fauvel, M., Chanussot, J., Benediktsson, J.A., and Sveinsson, J.R., 2008. Spectral and spatial classification of hyperspectral data using SVMs and morphological profiles. IEEE Transactions on Geoscience and Remote Sensing, 46, 38043814.

Gonzalez, R., and Woods, R., 2002. Digital Image Processing. 2nd ed. Englewood Cliffs, NJ: Prentice-Hall.

Huang, X., andZhang,L., 2011. A multidirectional and multiscale morphological index for automatic building extraction from mutispectral GeoEye-1 imagery.Photogrammetric Engineering and Remote Sensing,77, 721-732.

Negri, R.G., Dutra, L.V., andSant'Anna, S.J.-S., 2014. An innovative support vector machine based method for contextual image classification.ISPRS Journal of Photogrammetry and Remote Sensing,87, 241-248.

Paneque-Gálvez, J., Mas, J.-F., Moré, G., Cristóbal, J., OrtaMartínez, M., andLuz,A.C., 2013. Enhanced land use/cover classification of heterogeneous tropical landscapes using support vector machines and textural homogeneity.International Journal of Applied Earth Observation and Geoinformation, 23, 372-383.

Shackelford, A.K., and Davis, C.H., 2003. A combined fuzzy pixel-based and object-based approach for classification of high-resolution multispectral data over urban areas. IEEE Transactions on Geoscience and Remote Sensing, 41, 23542363.

Soille, P., 2003. Morphological Image Analysis. 2nd ed. Berlin, Germany: Springer-Verlag.

Tarabalka, Y., Chanussot, J., and Benediktsson, J.A., 2010. Segmentation and classification of hyperspectral images using minimum spanning forest grown from automatically selected markers. IEEE Translation System, Man, Cybern. B, Cybern., 40(5), 1267-1279.

Tarabalka, Y., Tilton, J.C., Benediktsson, J.A., andChanussot,J.A., 2011. Marker-Based Approach for the Automated Selection of a Single Segmentation From a Hierarchical Set of Image Segmentations.IEEE Journal of Selected Topics in Applied Earth Observations and Remote Sensing.

Tilton, J., 2003. Analysis of hierarchically related image segmentations.in Proc. IEEE Workshop Adv. Tech. Anal. Remotely Sensed Data, 60-69.

Wu, T.-F., Lin, C.-J., andWeng, R.C., 2004. Probability estimates for multiclass classification by pairwise coupling.J. Machine Learning Res., pp. 975-1005. 